\title{
16
}

\section{Remarks on the observability of nonlinear discrete time systems}

\author{
Francesca Albertini \\ Dipartimento di Matematica \\ Via Belzoni 7, 35131 Padova, Italy. Tel: +39(49)8275966. \\ e-mail: albertini@pdmat 1.math . unipd. it \\ Domenico D'Alessandro \\ Universita' di Padova, Dipartimento di Elettronica ed Informatica \\ Via Gradenigo 6a, 35100 Padova, Italy. Tel: +39(49)8287791. \\ e-mail: daless@maya.dei . unipd. it
}

\begin{abstract}
The observability of discrete time nonlinear systems is studied. Criteria of observability are given in terms of codistributions. This leads naturally to decompositions similar to the ones known in the continuous time case. Some observability properties of invertible systems are also investigated. In particular, it is shown that, under regularity hypotheses, the weaker notion of forward-backward observability is equivalent to the one of (forward) observability, for these systems.
\end{abstract}

Keywords

Discrete-time, nonlinear systems, observability.

\section{INTRODUCTION}

We deal with observability questions for nonlinear discrete-time systems of the form

$\begin{aligned} \Sigma \quad x(t+1) & =f(x(t), u(t)), \quad t=0,1,2, \ldots \\ y(t) & =h(x(t)) .\end{aligned}$

We consider single input single output systems, since the general case involves only notational changes. In $\Sigma$ we assume that $x(t) \in M, y(t) \in Y$ and $u(t) \in U$, with $M$ and $Y$ connected, second countable, Hausdorff, differentiable manifolds, of dimensions $n$ and 1 , respectively. We also assume that the control space $U$ is an open interval of $\mathbb{R}$, such that $0 \in U$. Such a system is said to be of class $C^{k}$, if the manifolds $M$ and $Y$ are of class $C^{k}$, and the functions $f: M \times U \rightarrow M$ and $h: M \rightarrow Y$, are of class $C^{k}$. We shall often use the abbreviated notation $f_{u}(x):=f(x, u)$. 
Definition 1 Two states $x_{1}, x_{2} \in M$ are said to be indistinguishable, and we write $x_{1} I x_{2}$, if for each sequence of controls, $u_{1}, \ldots, u_{j}$, with $j \geq 0$, we have

$h\left(f_{u_{j}} \circ \cdots \circ f_{u_{1}}\left(x_{1}\right)\right)=h\left(f_{u_{j}} \circ \cdots \circ f_{u_{1}}\left(x_{2}\right)\right)$.

Analogously $x_{1}, x_{2}$ are said to be $k$-indistinguishable, and we write $x_{1} I^{k} x_{2}$, if the previous condition holds for each $0 \leq j \leq k$.

Definition 2 One state $x_{0}$ is said to be observable ( $k$-observable), if, for each $x_{1} \in M$, $x_{0} I x_{1}\left(x_{0} I^{k} x_{1}\right)$ implies $x_{0}=x_{1}$.

Definition 3 One state $x_{0}$ is said to be locally observable ( $k$-observable), if there exists a neighborhood $W_{x_{0}}$ of $x_{0}$, such that, for each $x_{1} \in W_{x_{0}}, x_{0} I x_{1}\left(x_{0} I^{k} x_{1}\right)$ implies $x_{0}=x_{1}$.

Definition 4 A system $\Sigma$ is (locally) ( $k$-) observable, if each state $x \in M$ enjoys this property.

In the following, if we say that $x_{e}$ is an equilibrium point, we always mean that $f\left(x_{e}, 0\right)=$ $x_{e}$. We say that a subset of $M$ is generic if its complement is contained in a proper analytic subset of $M$. Given a set $L$ of $C^{\infty}$ functions, defined on $M$, we shall denote by $d L$ the codistribution spanned by all the differentials of these functions. By definition, these are exact differentials.

A previous study on the observability of discrete time nonlinear systems can be found in Nijmeijer (1982), where the case of systems without controls is considered. The use of the differential geometric concepts of invariant distributions and codistributions (see below), in the discrete time setting, is introduced in Monaco and Normand-Cyrot (1986). The classical paper Hermann and Krener (1977) deals with questions of nonlinear observability in the continuous time context. A complete treatment of this case is given in the books Isidori (1989) and Nijmeijer and Van der Schaft (1990).

\section{OBSERVABILITY CRITERIA}

From now on, assume that a $C^{\infty}$ system $\Sigma$ is given. Define

$\Theta=\left\{h\left(f_{u_{j}} \circ \cdots \circ f_{u_{1}}(\cdot)\right) \mid u_{1}, \ldots, u_{j} \in U^{j} j \geq 0\right\}$.

The previous set of functions will become the main object of our study. The following result holds:

Theorem 5 If dim $d \Theta\left(x_{0}\right)=n$, then $x_{0}$ is a locally observable state for $\Sigma$. Conversely if $\Sigma$ is locally observable, then there exists an open subset of $M$, where this condition is verified. If in addition the system is analytic and locally observable, then this condition is verified in a generic subset of $M$.

Proof. Assume $\operatorname{dim} d \Theta\left(x_{0}\right)=n$. Then, there exist $n$ functions in $\Theta, H_{i}(\cdot):=h\left(f_{u_{j i}^{i}} \circ \cdots \circ\right.$ $\left.f_{u_{1}^{i}}(\cdot)\right), i=1, \ldots, n$, whose differentials are linearly independent at $x_{0}$. By continuity, they 
also are independent in a neighborhood $W_{x_{0}}$ of $x_{0}$. Therefore, $H_{i}(\cdot), i=1, \ldots, n$, define a smooth mapping from $M$ to $Y^{n}$, which, restricted to $W_{x_{0}}$, is injective. If, for $x_{1} \in W_{x_{0}}$, it is $x_{1} I x_{0}$, in particular, for all $i=1, \ldots, n$, it must hold $H_{i}\left(x_{0}\right)=H_{i}\left(x_{1}\right)$. By the injectivity of $H_{i}(\cdot), i=1, \ldots, n$, it follows that $x_{0}=x_{1}$.

For the converse implication, assume that $\Sigma$ is locally observable and it does not exist an open subset of $M$ where $\operatorname{dim} d \Theta(x)=n$, which is equivalent to say that $\operatorname{dim} d \Theta(x)<n$ for all $x \in M$. Let $r=\max _{x \in M} \operatorname{dim} d \Theta(x)(<n)$, and choose $x_{0} \in M$, such that $\operatorname{dim} d \Theta\left(x_{0}\right)=$ $r$. By continuity, there exists an open neighborhood $W_{x_{0}}$ of $x_{0}$, such that $\operatorname{dim} d \Theta(x)=r$, for all $x \in W_{x_{0}}$. Therefore there exist $H_{1}(\cdot), \ldots, H_{r}(\cdot)$, in $\Theta$, whose differentials in $W_{x_{0}}$ are linearly independent. We may take these functions, $H_{1}(\cdot), \ldots, H_{r}(\cdot)$, along with a set of complementary independent functions, as partial coordinates in $W_{x_{0}}$. Since every function in $\Theta$ only depends on the first $r<n$ coordinates, points in $W_{x_{0}}$, with the last $n-r$ coordinates equal, cannot be distinguished. This contradicts the hypothesis of the local observability of the system, and shows that there exists an open subset in $M$, such that $\operatorname{dimd} \Theta(x)=n$ is true. The last sentence of the theorem follows easily from the fact that, if the above is true in an open subset of $M$, and the system is analytic, then, it is true everywhere except for the set of zeros of an analytic function, namely an analytic set. It is therefore true in what we have called a generic subset of $M$.

If $d \Theta$ is constant dimensional in a neighborhood of $x_{0}$, then a stronger result about local observability can be derived (the result is easily proved by specializing to $x_{0}$ the proof of Theorem 5).

Corollary 6 Assume $d \Theta$ is constant dimensional in a neighborhood $W_{x_{0}}$ of $x_{0}$. Then, $x_{0}$ is locally observable if and only if $\operatorname{dimd} \Theta\left(x_{0}\right)=n$.

Remark 7 Analogous criteria can be given for local $k$-observability, by considering, in the previous statements, the following set of functions

$$
\Theta_{k}=\left\{h\left(f_{u_{j}} \circ \cdots \circ f_{u_{1}}(\cdot)\right) \mid u_{1}, \ldots, u_{j} \in U^{j} k \geq j \geq 0\right\} .
$$

The proofs follow the same lines as above.

Remark 8 In view of Corollary 6 , it is of interest to give criteria for the constant dimensionality of the codistribution $d \Theta$, in a neighborhood of a given point. This is also important because, as we will see in the sequel, it is possible, in this situation, to obtain a local state space decomposition (see Section 3 below). Moreover, under this assumption, it is also possible to prove the equivalence between forward and forward-backward observability for invertible systems (see Section 4 below). In the continuous-time setting, the class of functions, used to characterize observability, gives raise to a constant dimensional codistribution if analyticity and accessibility are verified (see Nijmeijer and Van der Schaft (1990) Proposition 3.38). This is not true in general for $d \Theta$ or $d \Theta_{k}$, in the discrete-time case. The following example illustrates this issue and clarifies what we mean for accessibility in our context. Later, we shall give a sufficient condition for local constant dimensionality of $d \Theta$ (see Proposition 13). 
Example 9 We recall (Jakubczyk and Sontag (1990) and Albertini and Sontag (1993)) that a point $x_{0} \in M$ is said to be forward accessible, if it is possible to reach from it an open subset of $M$. For the following system, 0 is a forward accessible equilibrium point

$$
\begin{aligned}
& x_{1}(t+1)=x_{1}(t)+x_{2}^{2}(t)+u^{2}(t) \\
& x_{2}(t+1)=x_{1}(t) \\
& y(t)=x_{1}(t)
\end{aligned}
$$

In fact, if $x_{1}(0)=0$, and $x_{2}(0)=0$, it is $x_{1}(2)=u^{2}(0)+u^{2}(1)$ and $x_{2}(2)=u^{2}(0)$. Therefore, it is possible to reach, in two steps, any point such that $x_{1} \geq 0, x_{2} \geq 0$. The codistribution $d \Theta$ has not constant dimension in a neighborhood of 0 . In fact, it is $d h(x)=[1,0], d h\left(f_{u_{1}}(x)\right)=\left[1,2 x_{2}\right]$, and for $j \geq 2$, defined $\bar{H}(\cdot):=h\left(f_{u_{j}} \circ \cdots \circ f_{u_{2}}(\cdot)\right)$, it is

$$
\begin{aligned}
& \frac{\partial h\left(f_{u_{j}} \circ \cdots \circ f_{u_{1}}(x)\right)}{\partial x_{2}}=\frac{\partial \bar{H}\left(f_{u_{1}}(x)\right)}{\partial x} \frac{\partial f_{u_{1}}(x)}{\partial x_{2}} \\
& =\left[\frac{\partial \bar{H}\left(f_{u_{1}}(x)\right)}{\partial x_{1}}, \frac{\partial \bar{H}\left(f_{u_{1}}(x)\right)}{\partial x_{2}}\right]\left[2 x_{2}, 0\right]^{T}=\frac{\partial \bar{H}\left(f_{u_{1}}(x)\right)}{\partial x_{1}} 2 x_{2} .
\end{aligned}
$$

This shows that $d \Theta$ has dimension 1 for any point such that $x_{2}=0$, and 2 elsewhere.

\section{STATE SPACE DECOMPOSITION}

The study of the observability for nonlinear discrete time systems, using standard differential geometric tools, leads naturally to consider the notion of invariant distribution, as a natural generalization of the concept of invariant subspace, used in the study of linear systems. This concept has been widely used in characterizing structural properties of nonlinear continuous time systems and its importance, in the study of the discrete time case, seems to have been first pointed out in Monaco and Normand-Cyrot (1984). We will use the dual concept of invariant codistribution to perform a state space decomposition, in a neighborhood of an equilibrium point. The theory very much resembles the continuous time one presented in Isidori (1989).

Recall that, given a covector field $\omega$ and a mapping $f(\cdot)$, on a manifold $M, f^{*} \omega:=$ $\omega(f(\cdot)) \frac{\partial f}{\partial x}(\cdot)$. Notice that, in particular, if $\omega$ is an exact differential, i.e. $\omega=d \lambda$, for a function $\lambda(\cdot)$, it is $f^{*} \omega=d(\lambda \circ f(\cdot))$. We have the following definition:

Definition 10 A codistribution $\Omega$ is said to be invariant under $f_{u}$, if

$f_{u}^{*} \Omega \subseteq \Omega, \quad$ for each $u \in U$

Theorem 11 Suppose that there exists a constant dimensional $f_{u}$ - invariant codistribution of dimension $r$, containing $d h$, and spanned by exact differentials, in a neighborhood 
of an equilibrium point $x_{e}$. Then there exists a coordinate change, such that for $(x, u)$ in a suitable neighborhood $\tilde{W}_{x_{e}} \times U_{0}$ of $\left(x_{e}, 0\right)$, the system $\Sigma$ reads as

$$
\begin{aligned}
z_{1}(t+1) & =\hat{f}\left(z_{1}(t), z_{2}(t), u(t)\right), \quad t=0,1,2, \ldots \\
\Sigma^{\prime} z_{2}(t+1) & =\hat{f}\left(z_{2}(t), u(t)\right) \\
y(t) & =\hat{h}\left(z_{2}(t)\right)
\end{aligned}
$$

where $z_{1}$ and $z_{2}$ have dimensions $n-r$ and $r$ respectively.

Proof. Under the stated hypotheses, there exist $r$ functions $\lambda_{1}(\cdot), \ldots, \lambda_{r}(\cdot)$ on $M$, such that $d \lambda_{1}\left(x_{e}\right), \ldots, d \lambda_{r}\left(x_{e}\right)$ form a basis of $\Omega\left(x_{e}\right)$. These functions, along with a complementary set of $n-r$ linearly independent functions, give a coordinate change, $T(x)$, in a neighborhood $W_{x_{e}}$ of $x_{e}$. We write $\hat{h}(\cdot), \hat{f}(\cdot), \hat{\lambda}_{i}(\cdot)$, for $h(\cdot), f(\cdot)$ and $\lambda_{i}(\cdot), i=1, \ldots, r$, respectively, in these coordinates. In particular, if $z=T(x)$ denotes the new coordinates, we can as well assume that $\hat{\lambda}_{i}=z_{n-r+i}, i=1, \ldots, r$. Notice that, since $d h$ is in $\Omega, \hat{h}(\cdot)$ only depends on the last $r$ coordinates. By continuity, we can choose a neighborhood $\tilde{W}_{x_{e}} \times U_{0}$ of $\left(x_{e}, 0\right)$, such that $f_{u}(x) \in W_{x_{e}}$, for all pairs $(x, u) \in \tilde{W}_{x_{e}} \times U_{0}$. Choosing $x$ and $u$ in this way, and remembering that, by the invariance property, $d \lambda_{i}\left(f_{u}\right)$, for $i=1, \ldots, r$, is still in $\Omega$, we also have

$\mathbf{0}=\frac{\partial}{\partial z_{j}} \hat{\lambda}_{i}\left(\hat{f}_{u}(\cdot)\right)=\frac{\partial \hat{f}_{u}^{n-\tau+i}}{\partial z_{j}}(\cdot)$

$i=1, \ldots, r, j=1, \ldots, n-r$. This shows that the last $r$ components of $\hat{f}(\cdot)$ are independent of the first $n-r$ components of $z$. Therefore, locally the system can be written in the form $\Sigma^{\prime}$.

If $d \Theta$ is constant dimensional in a neighborhood of $x_{\varepsilon}$, it is a good candidate to be used to perform the change of coordinates described above. $d \Theta$ is, in fact, $f_{u}$-invariant, it is spanned by exact differentials, and it contains $d h$. Moreover $d \Theta$ is the smallest codistribution which enjoys these properties. The proof of this fact follows the same lines of the one given for the observability codistribution in the continuous time case Isidori (1989). The only modification consists in replacing the definition of invariance, given in the continuous time context, with the one given here. Notice that the only if part of Corollary 6 also holds if we consider an arbitrary constant dimensional codistribution which properly contains $d \Theta$. In view of this fact, in the hypotheses of Theorem 11, since $\Omega$ must contain $d \Theta$, if $r<n, x_{e}$ is not locally observable.

\section{PROPERTIES OF INVERTIBLE SYSTEMS}

In this section, we will deal with a particular class of discrete time nonlinear systems.

Definition 12 A system $\Sigma$ is said to be invertible, if for all $u \in U$, the function $f_{u}: M \rightarrow$ $M$, is a diffeomorphism (we denote by $f_{u}^{-1}$ the inverse function of $f_{u}$ ). 
Invertible systems arise, for example, when a continuous-time model is controlled under digital control, via sampling. Further motivations for the study of this class of systems are given in Jakubczyk and Sontag (1990). For invertible systems, it is possible to define an inverse system by

$\begin{array}{ll}\Sigma^{-} \quad x(t+1) & =f^{-1}(x(t), u(t)), \quad t=0,1,2, \ldots \\ y(t) & =h(x(t)) .\end{array}$

Using this system one can define backward indistinguishability and observability, following the same lines of Definitions 1-4. It is also possible to define (see example 9 and Jakubczyk and Sontag (1990), Albertini and Sontag (1993)) backward accessibility. These definitions extend to forward-backward indistinguishability, observability and accessibility in an obvious manner. We state now a sufficient condition for the constant dimensionality of the codistribution $d \Theta$ defined in (1)

Proposition 13 Consider an invertible system $\Sigma$ and an equilibrium point $x_{e}$. Assume that the following rank condition is verified

$$
\sup _{j \geq n}\left\{\left.\operatorname{rank} \frac{\partial}{\partial u}\right|_{u_{1}=\ldots=u_{j}=0} f_{u_{j}} \circ \cdots \circ f_{u_{1}}\left(x_{e}\right)\right\}=n .
$$

Then, the codistribution $d \Theta$ is constant dimensional, in a neighborhood of $x_{e}$.

Proof. Using the rank condition (2), it is easy to show that there exist an open subset of $M, F\left(x_{e}\right)$, which contains $x_{e}$, and such that each point of $F\left(x_{e}\right)$ can be reached by $x_{e}$. Also it can be shown that there exists an open subset $B\left(x_{e}\right)$, which contains $x_{e}$, and such that from any point in it, it is possible to reach $x_{e}$. (The proof of these facts follows from the one of the accessibility criterion given in Proposition 2.3 of Jakubczyk and Sontag (1990).) We consider the set $L\left(x_{e}\right)=B\left(x_{e}\right) \cap F\left(x_{e}\right) . L\left(x_{e}\right)$ is an open set and contains $x_{c}$. Moreover, for a point $x_{1}$ in $L\left(x_{c}\right)$, there exist two sequences of controls such that

$x_{1}=f_{u_{j}^{F} F} \circ \cdots \circ f_{u_{1}^{F}}\left(x_{e}\right), \quad$ and $\quad x_{e}=f_{u_{j B}^{B}} \circ \cdots \circ f_{u_{1}^{B}}\left(x_{1}\right)$.

Consider now $k_{F}$ functions, $h^{i}(\cdot), i=1, \ldots, k_{F}$, such that $d h^{i}\left(x_{1}\right), i=1, \ldots, k_{F}$, form a basis for $d \Theta\left(x_{1}\right)$. The functions $h^{i}\left(f_{u_{j}^{F}} \circ \cdots \circ f_{u_{1}^{F}}(\cdot)\right), i=1, \ldots, k_{F}$ are in $\Theta$; moreover, using (3) (left hand side),

$d h^{i}\left(f_{u_{j F}^{F}} \circ \cdots \circ f_{u_{1}^{F}}\left(x_{e}\right)\right)=d h^{i}\left(x_{1}\right) \frac{\partial f_{u_{j F}^{F}} \circ \cdots \circ f_{u_{1}^{F}}\left(x_{e}\right)}{\partial x}$.

By the invertibility of $f_{u_{j}^{F}} \circ \cdots \circ f_{u_{1}^{F}}(\cdot), \frac{\partial f_{u_{j}^{F}} \circ \cdots \circ f_{u_{1}^{F}}\left(x_{e}\right)}{\partial x}$ is nonsingular, so, by the linear independence of $d h^{i}\left(x_{1}\right)$, we have that $d h^{i}\left(f_{u_{j^{F}}^{F}} \circ \cdots \circ f_{u_{1}^{F}}\right)\left(x_{e}\right)$ also are linearly independent. Therefore they can be included in a basis of $d \Theta\left(x_{e}\right)$, and this shows that

$\operatorname{dim} d \Theta\left(x_{1}\right) \leq \operatorname{dimd} \Theta\left(x_{e}\right)$. 
Analogously, if we choose a set of functions $h^{i}(\cdot), i=1, \ldots, k_{B}$, such that $d h^{i}\left(x_{e}\right)$ form a basis for $d \Theta\left(x_{e}\right)$, we have that $h^{i}\left(f_{u_{j}^{B}} \circ \cdots \circ f_{u_{1}^{B}}(\cdot)\right), i=1, \ldots, k_{B}$, is still in $\Theta$. By the invertibility of $f_{u_{j}^{B} B} \circ \cdots \circ f_{u_{1}^{B}}(\cdot)$ and using (3) (right hand side), we have, as above,

$\operatorname{dimd} \Theta\left(x_{e}\right) \leq \operatorname{dimd} \Theta\left(x_{1}\right)$.

Combining (4) and (5), we have that $\Theta$ is constant dimensional in $L\left(x_{e}\right)$.

We conclude showing that, under regularity hypotheses, locally forward observability (l.f.o) and locally forward-backward observability (l.f.b.o) are equivalent for equilibrium points of invertible systems.

Theorem 14 Let $\Sigma$ be an analytic invertible system, and $x_{e} \in M$ be an equilibrium point. Assume that $d \Theta$ has constant dimension in a neighborhood $W_{x_{e}}$ of $x_{e}$. Then, $x_{e}$ is l.f.o. if and only if it is l.f.b.o.

Proof. It is obvious that if $x_{e}$ is l.f.o, it is also l.f.b.o. Conversely, assume that $x_{e}$ is not l.f.o. Under the stated hypotheses and using Corollary 6 , we know that $\operatorname{dim} d \Theta(x)=r<n$, in a neighborhood $W_{x_{e}}$ of $x_{\mathrm{e}}$. We can therefore perform a change of coordinates as in Theorem 11. System $\Sigma$ reads as $\Sigma^{\prime}$, as long as, $x$ is in a suitable neighborhood $\tilde{W}_{x_{e}} \subseteq W_{x_{e}}$ and $u$ is in a suitable neighborhood $U_{0}$ of 0 . It is straightforward to verify that also the inverse system $\Sigma^{-}$can be put in a triangular form, again for $x \in \tilde{W}_{x_{e}}$, and $u \in U_{0}$.

Assume, by contradiction, that $x_{e}$ is l.f.b.o, and let $V_{x_{e}} \subseteq \tilde{W}_{x_{e}}$, be a neighborhood of $x_{e}$, where the l.f.b.o. holds. Then choose any $\bar{x} \in V_{x_{e}}$ such that, in the $z$-coordinate, the last $r$ components of $x_{e}$ and of $\bar{x}$ are equal. Since $\bar{x} \in V_{x_{e}}$, there exists $k, \tilde{u}_{1}, \ldots, \tilde{u}_{k} \in U$, $\epsilon_{1}, \ldots, \epsilon_{k}$, with $\epsilon_{i}= \pm 1, i=1, \ldots, k$, such that:

$h\left(f_{\tilde{u}_{k}}^{\epsilon_{k}} \circ \cdots \circ f_{\tilde{u}_{1}}^{\epsilon_{1}}\left(x_{e}\right)\right) \neq h\left(f_{\tilde{u}_{k}}^{\epsilon_{k}} \circ \cdots \circ f_{\tilde{u}_{1}}^{\epsilon_{1}}(\bar{x})\right)$.

By continuity, there exists $\tilde{U}_{0} \subseteq U_{0}$ neighborhood of 0 such that, for $i=1, \ldots, k$ :

$u_{1}, \ldots, u_{i} \in \tilde{U}_{0} \Rightarrow f_{u_{i}}^{\epsilon_{i}} \circ \cdots \circ f_{u_{1}}^{\epsilon_{1}}\left(x_{e}\right) \in \tilde{W}_{x_{e}}$, and $f_{u_{i}}^{\epsilon_{i}} \circ \cdots \circ f_{u_{1}}^{\epsilon_{1}}(\bar{x}) \in \tilde{W}_{x_{e}}$.

From (6), and by analyticity, there exists $\bar{u}_{1}, \ldots, \bar{u}_{k}$, with $\bar{u}_{i} \in \tilde{U}_{0}$, such that

$h\left(f_{\bar{u}_{k}}^{\epsilon_{k}} \circ \cdots \circ f_{\bar{u}_{1}}^{\epsilon_{1}}\left(x_{\epsilon}\right)\right) \neq h\left(f_{\tilde{u}_{k}}^{\epsilon_{k}} \circ \cdots \circ \int_{\bar{u}_{1}}^{\epsilon_{1}}(\bar{x})\right)$.

However, since $\bar{u}_{i} \in \tilde{U}_{0}$, equation (7) implies that, $\forall i=1, \ldots, k, f_{\bar{u}_{i}}^{\epsilon_{i}} \cdots \cdots \circ f_{\bar{u}_{1}}^{\epsilon_{1}}\left(x_{e}\right)=$ $x_{e}^{i} \in \tilde{W}_{x_{e}}$ and also $f_{u_{i}}^{\epsilon_{i}} \circ \cdots \circ f_{\bar{u}_{1}}^{\epsilon_{1}}(\bar{x})=\bar{x}^{i} \in \tilde{W}_{x_{e}}$. Thus, by the triangular form, in the $z$-coordinate, the last $r$ components of $x_{e}^{i}$ and of $\bar{x}^{i}$ are equal. So, also $h\left(x_{e}^{k}\right)=h\left(\bar{x}^{k}\right)$, which contradicts equation (8).

\section{REFERENCES}

Albertini, F. and Sontag, E.D. (1994) Discrete-time transitivity and accessibility: analytic systems. SIAM J. Control and Opt., 31, 1599--622. 
Hermann, R. and Krener, A.J. (1977) Nonlinear controllability and observability. IEEE Trans. Automat. Control., AC-22, 728-40.

Isidori, A. (1989) Nonlinear Control Systems. 2nd edition, Springer Verlag, Berlin.

Jakubczyk, B. and Sontag, E.D. (1990) Controllability of nonlinear discrete-time systems: A Lie-algebraic approach, SIAM J. Control and Opt., 28, 1-33.

Monaco, S. and Normand-Cyrot, D. (1984) Invariant distributions for discrete time nonlinear systems. Systems and Control Letters, 5, 191-6.

Nijmeijer, H. (1982) Observability of autonomous discrete time non-linear systems: a geometric approach. Int.J.Control, 36, 867-74.

Nijmeijer, H. and Van der Schaft, A. J. (1990) Nonlinear Dynamical Control Systems. Springer-Verlag, New York. 